\title{
Picture quiz 2
}

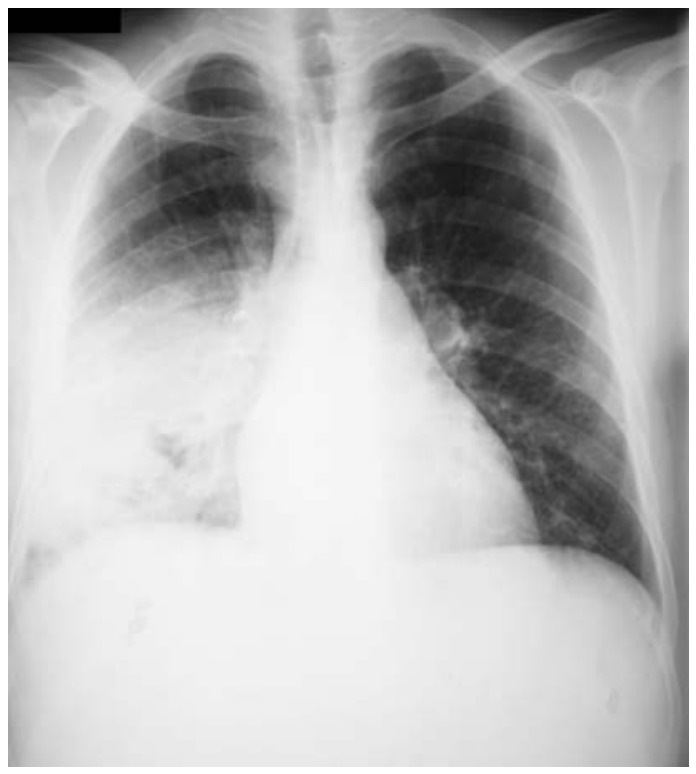

Image 1: PA chest radiograph.

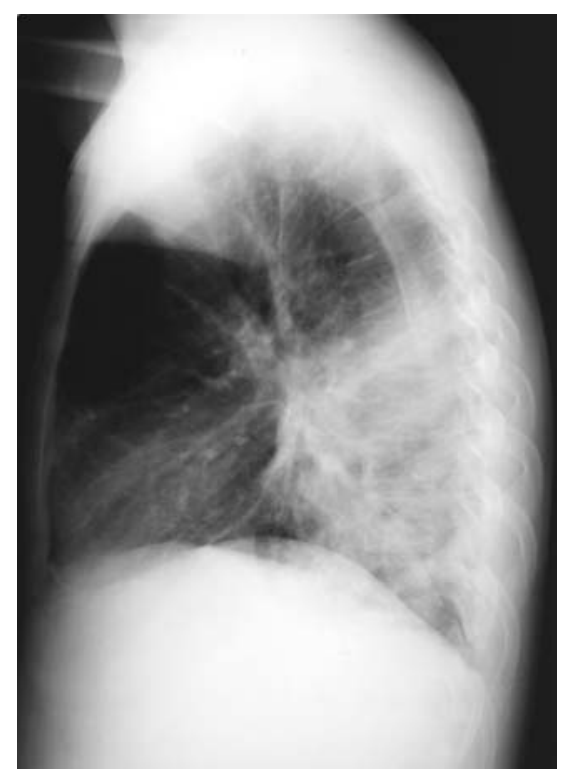

Image 2: Right lateral chest radiograph.

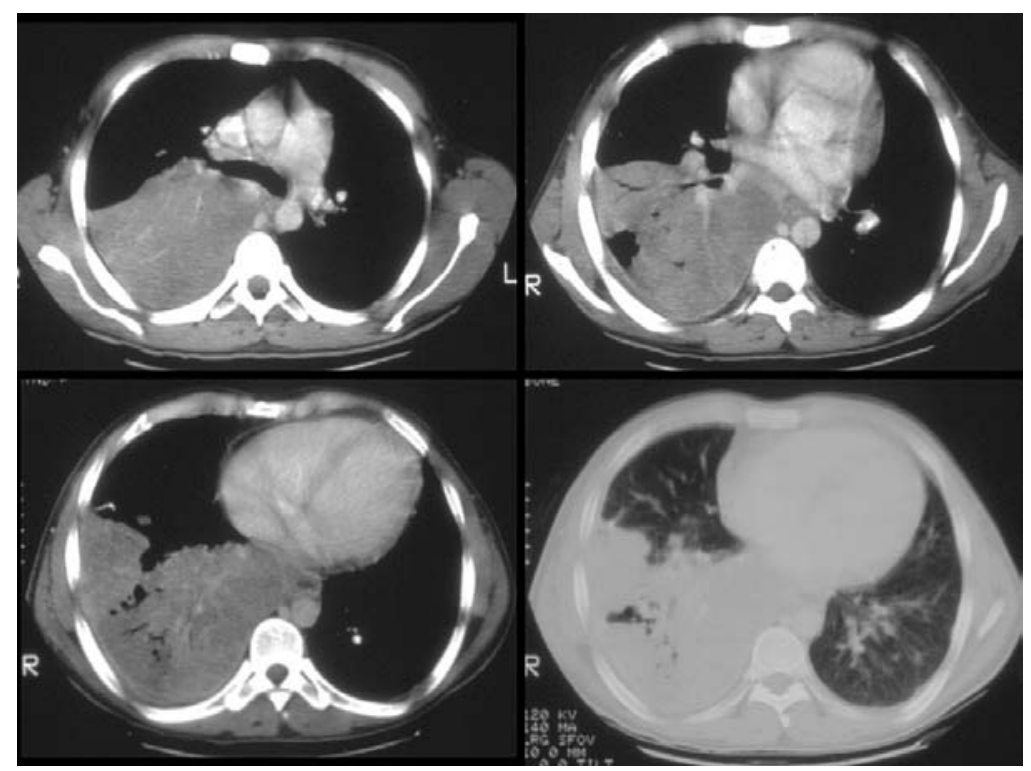

Image 3 : Post-contrast CT chest - slices 1, 2, 3 in mediastinal window and slice 4 in lung window setting.

\section{Question}

A 29-year old man admitted to the hospital after failed therapy for outpatient pneumonia. What are your observations? What is your diagnosis?

\section{Answer}

Diagnosis: Right lower lobe, lobar pneumonia : Pseudomonas cepacia. 
Description: $\quad$ Image 1 and Image 2. PA and lateral chest radiographs demonstrate extensive right lower lobe airspace consolidation. The homogeneity and lack of volume loss are consistent with the "lobar" pattern of pneumonia.

Image 3. Contrast enhanced chest CT (mediastinal and lung window) demonstrates uniform airspace consolidation of the right lower lobe with patent central bronchi (slice 2). Focal early cavitation is seen peripherally (slices 2, 3 and 4).

Differential diagnosis: Other lobar pneumonias, drowned lung, bronchioloalveolar cell carcinoma, lymphoma.

Discussion:

Lobar consolidation produces homogeneous parenchymal opacity. Although initially nonsegmental, advanced disease affects entire segments or lobes, accounting for the term "lobar" pneumonia. Lung volume is maintained or even expanded because of the extensive inflammatory response and alveolar exudates associated with lobar pneumonias.

Five percent of lobar pneumonias are caused by organisms other than Streptococcus pneumoniae and Klebsiella pneumoniae. The Presence of cavitation in this case suggests aetiologies other than $S$. pneumoniae.

\section{Reference}

Hansell DM, Dee P. Infections of the lungs and pleura. In: Armstrong Peter, Wilson Alan G, Dee Paul, Hansell David M, eds. Imaging of Diseases of the Chest. 3rd ed. London: Harcourt Publishers Limited, 2000:163-253.

AL Chang,Consultant radiologist; e-mail:<ailee32@yahoo.co.uk>.

\section{Processing fee}

A processing fee is charged from authors at the time of submission of an article to the $C M J$. For authors resident overseas, the processing fee is as follows.
- SAARC countries USD 20
- UK and Europe USD 30
- All other countries USD 35

Payment is in US Dollars only, and by Bank Draft, or by certified cheque, or other reliable transfer, written in favour of "Ceylon Medical Journal A/C 0030330568 CU” and crossed "Account Payee Only".

Editors, Ceylon Medical Journal, Wijerama House, 6, Wijerama Mawatha, Colombo 7, Sri Lanka. Inquiries: e-mails: <hjdes@sltnet.lk> or <colvin_goonaratna@yahoo.com>. 